\title{
Dual Inhibition of Klebsiella pneumoniae and Pseudomonas aeruginosa Iron Metabolism using Gallium Porphyrin and Gallium Nitrate.
}

Seoung-ryoung Choi ${ }^{1}$, Bradley E. Britigan ${ }^{1,2,3}$, and Prabagaran Narayanasamy ${ }^{1, *}$

${ }^{1} 985900$ Nebraska Medical Center, Department of Pathology and Microbiology, College of Medicine, University of Nebraska Medical Center, Omaha, Nebraska, USA, 68198.

${ }^{2}$ Department of Internal Medicine, College of Medicine, University of Nebraska Medical Center, Omaha, Nebraska, USA.

${ }^{3}$ Department of Internal Medicine and Research Service, Veterans Affairs Medical CenterNebraska Western Iowa, Omaha, Nebraska, USA.

PN - p.narayanasamy@unmc.edu

\section{List of Table}

Figure S1. Checkerboard assay of GaPP in combination with $\mathrm{Ga}\left(\mathrm{NO}_{3}\right)_{3}$ against bacteria in iron-

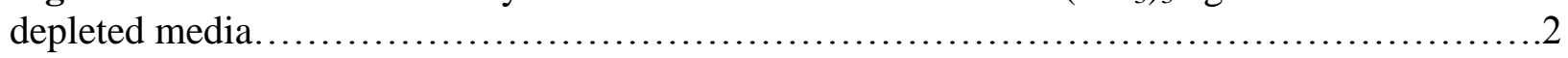

Figure S2. Checkerboard assay of $\mathrm{Ga}\left(\mathrm{NO}_{3}\right)_{3}$ in combination with antibiotic against $K$. $\begin{array}{llll}\text { pneumoniae } & \text { in } & \text { iron-depleted }\end{array}$ media. .2

Figure S3. Checkerboard assay of GaPP with antibiotic against K. pneumoniae in iron-depleted BM2 media. 3

Figure S4. Checkerboard assay of $\mathrm{Ga}\left(\mathrm{NO}_{3}\right)_{3}$ with antibiotic against $P$. aeruginosa in irondepleted BM2 media. .3

Figure S5. Checkerboard assay of GaPP with antibiotic against $P$. aeruginosa in iron-depleted

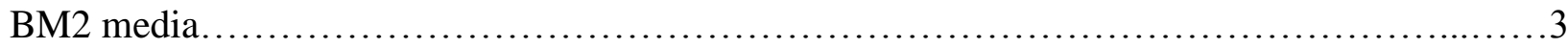

Figure S6. Three-drug checkerboard assay of $P$. aeruginosa and $K$. pneumoniae in iron-depleted BM2 media..................................................................4

Figure S7. $K$. pneumoniae growth curve in the presence of FAC, hemin, or both.... . .4

$\begin{array}{llllll}\text { Figure } & \text { S8. } & \text { Time-kill } & \text { assay } & \text { resistant } & P \text {. }\end{array}$ aeruginosa.

Figure S9. Survival of C.elegans infected with $P$. aeruginosa or $K$. pneumoniae . .5 

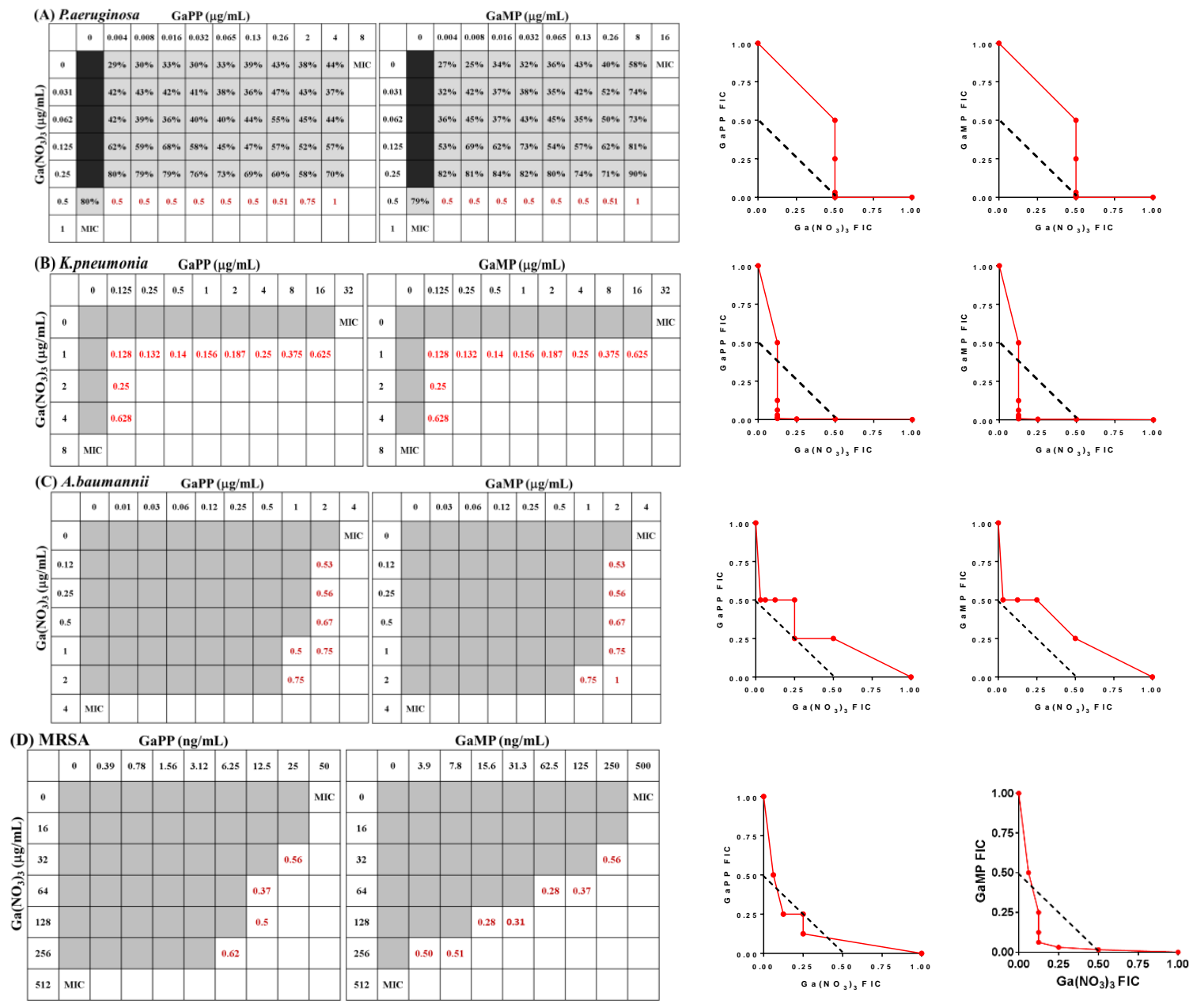

Figure S1. Checkerboard assay of $\mathrm{GaPP}$ in combination with $\mathrm{Ga}\left(\mathrm{NO}_{3}\right)_{3}$ against different bacteria in iron-depleted media.
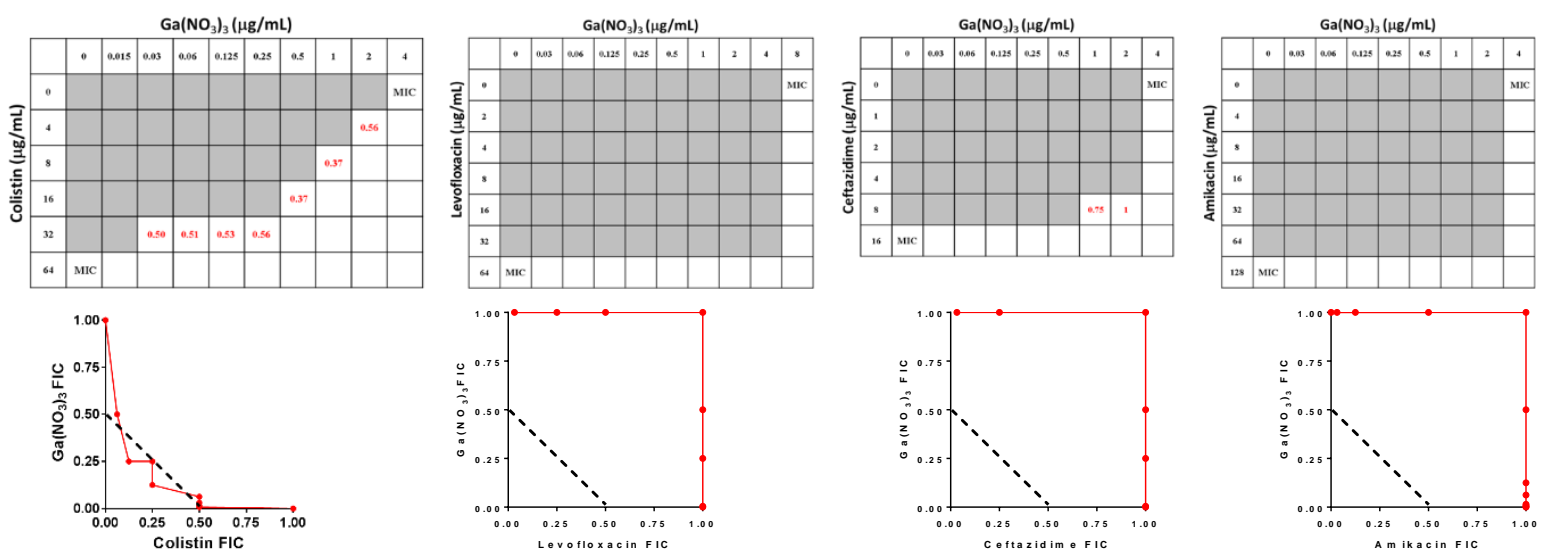

Figure S2. Checkerboard assay of $\mathrm{Ga}\left(\mathrm{NO}_{3}\right)_{3}$ in combination with conventional antibiotics against $K$. pneumoniae in iron-depleted BM2 media. 

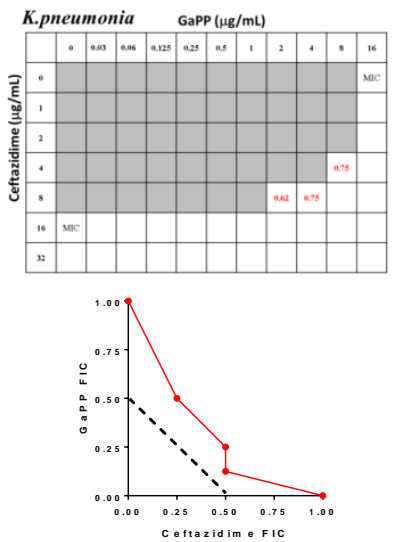
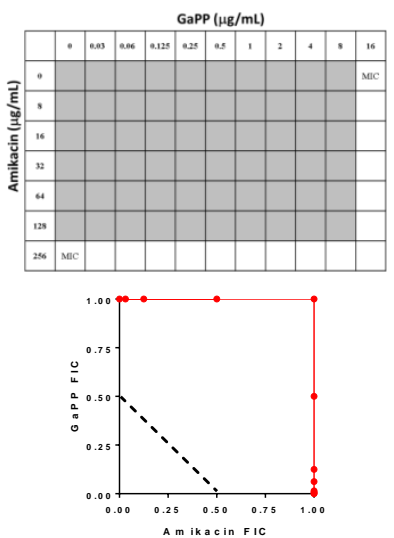
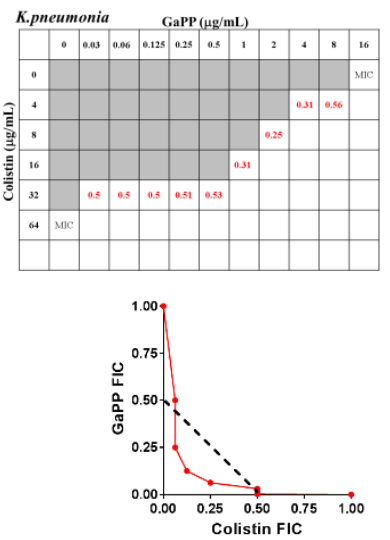
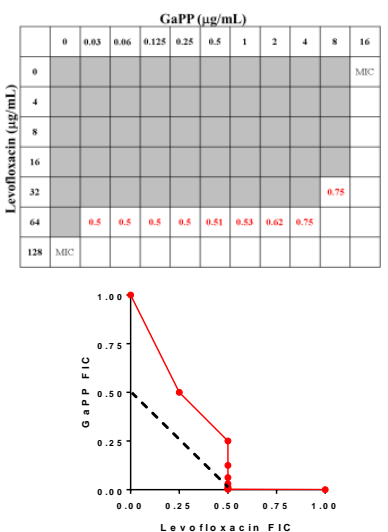

Figure S3. Checkerboard assay of GaPP in combination with conventional antibiotics against $K$. pneumoniae ATCC BAA 1705 in iron-depleted BM2 media.
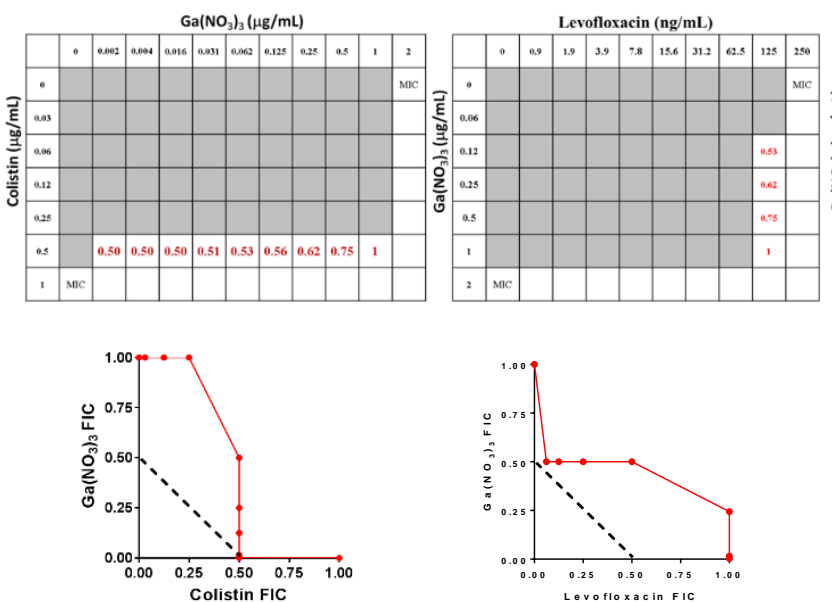
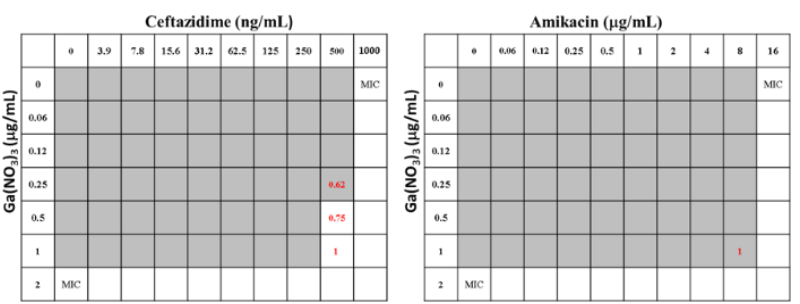

Figure S4. Checkerboard assay of $\mathrm{Ga}\left(\mathrm{NO}_{3}\right)_{3}$ in combination with conventional antibiotics against $P$. aeruginosa PA103 in iron-depleted BM2 media.
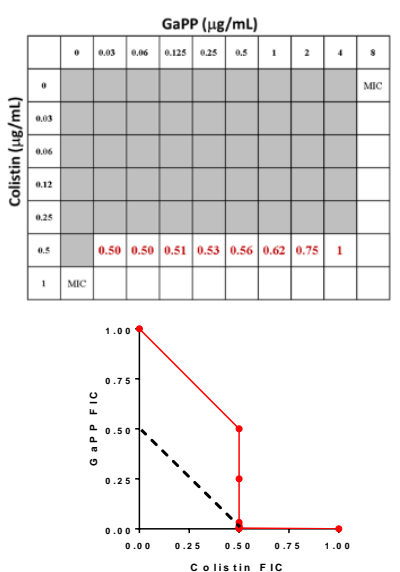
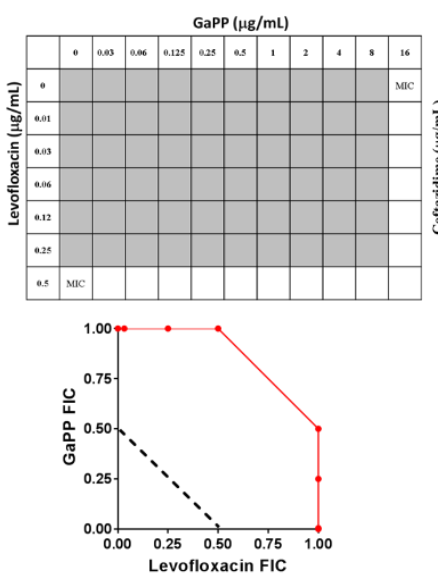
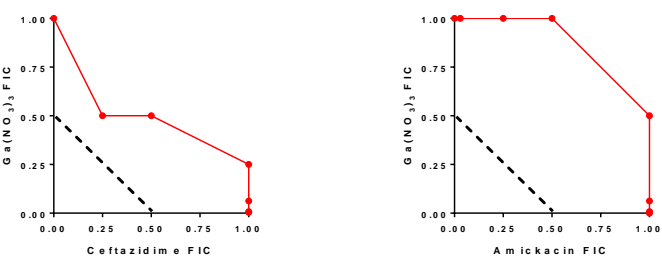

Figure S5. Checkerboard assay of GaPP in combination with conventional antibiotics against $P$. aeruginosa PA103 in iron-depleted BM2 media. 


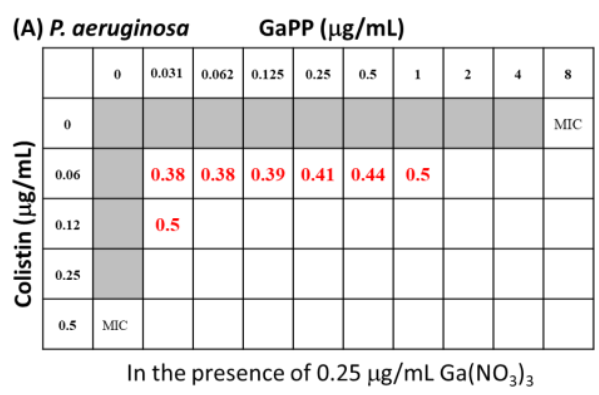

Figure S6. Three-drug checkerboard assay of $P$. aeruginosa PA103 and CRKP in iron-depleted BM2 media.

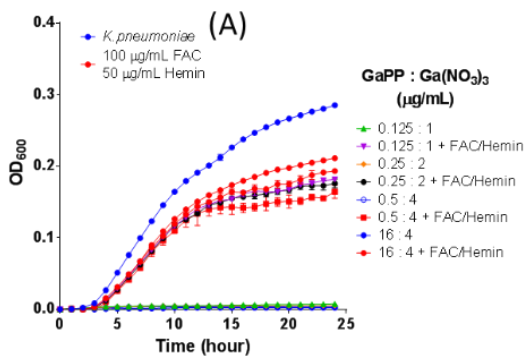

(D)

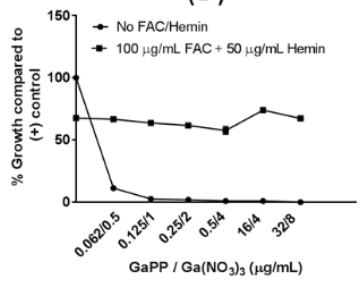

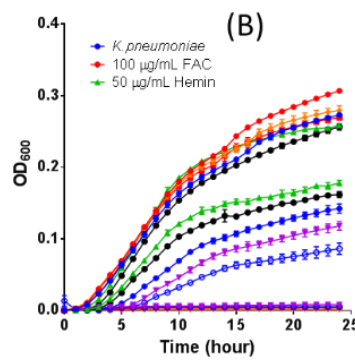

(E)

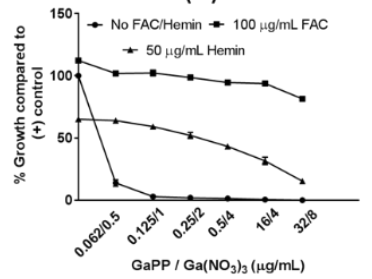

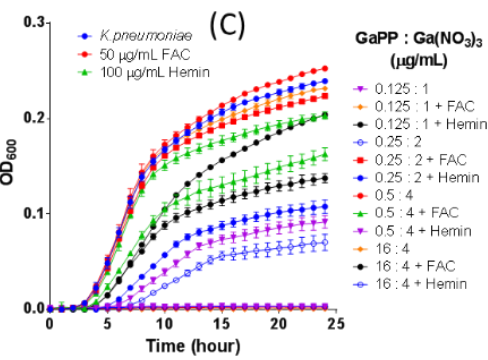

(F)

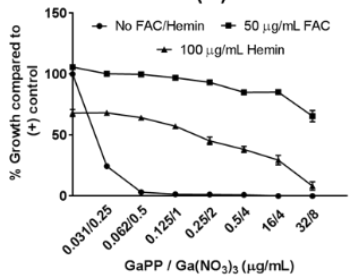

Figure S7. K. pneumoniae growth curve in the presence of FAC, hemin, or both. K. pneumoniae was cultured in iron-depleted BM2 media. CRKP growth (\% compared to untreated) at $24 \mathrm{~h}$ of incubation in the presence of FAC, Hemin, or both. Graph (D) shows simplified \% growth compared to (+) control- (No Ga(III), FAC, and hemin) at $24 \mathrm{~h}$ of incubation from growth curves. Similarly, Graph (E) is derived from growth curves (B), and Graph (F) from (C) at 24 h. 

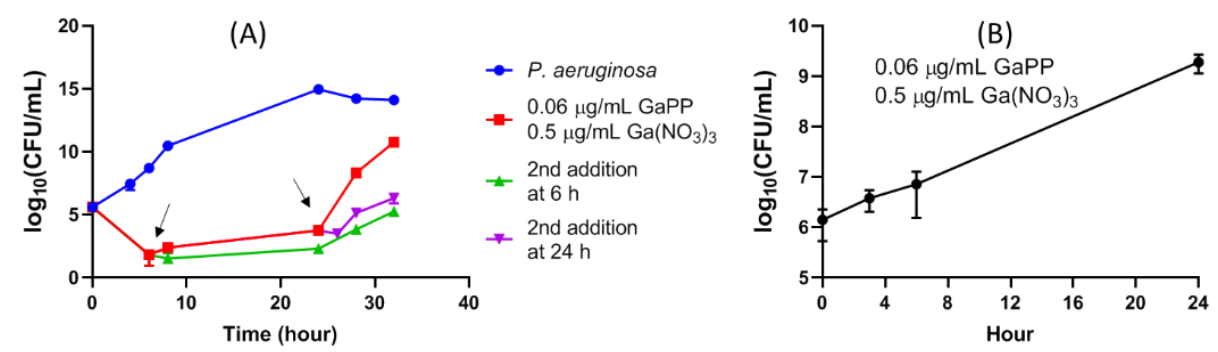

Figure S8. Time-kill assay of resistant $P$. aeruginosa. (A) Additional Ga(III) inhibitors were added at 6 h or 24 h of incubation. (B) Regrown P. aeruginosa from (A) was washed with BM2 medium and inoculated in the presence of $\mathrm{Ga}(\mathrm{III})$-based inhibitory compounds.
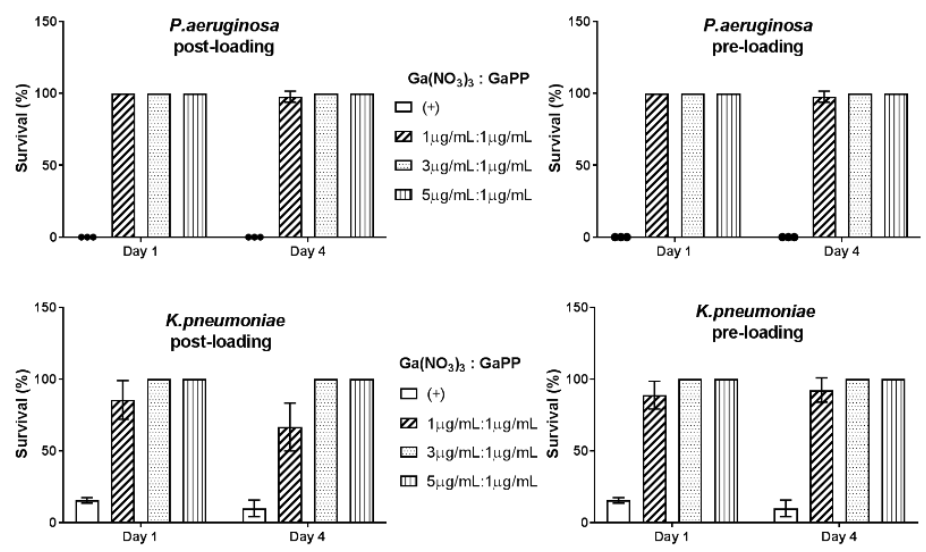

Figure S9. Survival of C. elegans infected with P. aeruginosa or K. pneumoniae. 